\title{
Conflits et reconfigurations socio-spatiales autour du sanglier. Des postures générales aux arrangements locaux
}

Conflicts, social and spatial changes about wild board. Stereotyped stances to local arrangements

\section{Coralie Mounet}

\section{(2) OpenEdition \\ Journals}

Édition électronique

URL : http://journals.openedition.org/economierurale/3367

DOI : 10.4000/economierurale.3367

ISSN : 2105-2581

\section{Éditeur}

Société Française d'Économie Rurale (SFER)

Édition imprimée

Date de publication : 1 mars 2012

Pagination : 79-95

ISSN : 0013-0559

Référence électronique

Coralie Mounet, "Conflits et reconfigurations socio-spatiales autour du sanglier. Des postures générales aux arrangements locaux », Économie rurale [En ligne], 327-328 I janvier-mars 2012, mis en ligne le 01 mars 2014, consulté le 02 mai 2019. URL : http://journals.openedition.org/ economierurale/3367 ; DOI : 10.4000/economierurale.3367 


\section{Conflits et reconfigurations socio-spatiales autour du sanglier}

\section{Des postures générales aux arrangements locaux}

Coralie MOUNET • Cemagref, UR Développement des territoires montagnards, Saint-Martin-d'Hères coraliemounet@hotmail.com

A utrefois consacrés principalement à 'agriculture, les territoires ruraux subissent aujourd'hui des mutations tant sociales qu'environnementales (Perrier-Cornet, 2002). Sociales, avec une activité agricole qui ne fait plus à elle seule le rural : les territoires sont aujourd'hui investis par de multiples autres enjeux qu'ils soient environnementaux, cynégétiques, récréatifs, résidentiels. Environnementales, avec la progression numérique et spatiale d'une grande faune sauvage (Mauz, 2006b) qui, profitant des retombées actuelles d'une politique de protection environnementale, se développe et colonise de nouveaux territoires. Les agriculteurs voient ainsi leur maîtrise et leur emprise spatiales empiétées à la fois par des hommes, dont les cadres de pensée du sauvage et de l'environnement diffèrent fortement des leurs (Micoud, 1993) et par les animaux sauvages, dont les incursions dans l'espace domestique se multiplient (Mounet, 2006).

La chasse au sanglier ${ }^{1}$ met en scène ces questionnements sur la place sociale et spatiale des activités agricoles ou cynégétiques au sein des territoires ruraux ainsi que sur la répartition des espaces voués au sauvage ou au domestique. D'une part, les agriculteurs et les chasseurs sont de plus en plus distincts et ne partagent plus les mêmes intérêts ni les mêmes préoccupations. Le fossé se creuse entre des gestionnaires d'espèces sauvages, des «faiseurs de sauvage » et ceux qui, depuis plusieurs siècles, ont

1. Pour plus d'informations sur l'évolution générale des chasseurs de sanglier et de leurs pratiques cynégétiques, voir Raynal, 2004.

entrepris une domestication de la montagne (Mauz, 2006a). D'autre part, le développement des populations de sangliers a été tel, ces vingt dernières années, que de nouveaux territoires ont été colonisés, comme les zones montagnardes (Baubet, 1998). Or, le sanglier figure dans la liste des animaux « à problème » (Micoud et Bobbé, 2006) qu'il est bien difficile de gérer : il est la cible de plusieurs enjeux contradictoires, entre volonté de réduction des populations pour limiter les dégâts agricoles et volonté de conservation d'une population conséquente, pour permettre la pratique de la chasse.

Si les travaux de nombreux auteurs ${ }^{2}$ apportent des éclairages sur les conflits d'aménagement et d'environnement et de manière plus large sur les conflits d'usage, ils ne parviennent pas à couvrir les spécificités de ceux provoqués par cette confrontation d'humains et d'animaux qui n'ont pas ou plus coutume de se côtoyer. Ces derniers ne peuvent être en effet considérés comme des conflits d'environnement stricto sensu ou d'aménagement (Charlier, 1999) puisqu'il s'agit ici des retombées de la gestion de l'environnement sur les activités agricoles et non l'inverse. De plus, par leur mobilité et leur comportement, les animaux rendent les situations beaucoup plus complexes que celles provoquées par un aménagement : leurs spatialités ${ }^{3}$ propres

2. Notamment, Charlier (1999), Lecourt (2003), Melé et al., (2003), Dziedzicki (2001), Torre et al. (2005).

3. Entendue comme «l'ensemble des actions spatiales réalisées par les opérateurs d'une société » (Lussault, 2003). 
interrogent les territoires des hommes et créent des incertitudes. En outre, si les conceptions du territoire issues de la géographie humaine, enrichies des outils de la sociologie de l'action organisée, permettent de décrire les systèmes de relations entre acteurs, elles laissent peu de place aux non humains. L'aspect actif des animaux composant le milieu naturel est en effet bien souvent laissé de côté, tant par la géographie classique prenant l'espace et le milieu naturel comme support aux relations sociales que la géographie sociale et culturelle prenant l'espace comme un construit social.

L'étude d'animaux à problème nous a donc imposé d'apporter un regard particulier, mobilisant la théorie de l'acteur-réseau (Latour, 2006). La sociologie des collectifs accorde en effet des compétences sociales aux non humains, en leur reconnaissant une capacité à créer des liens sociaux. Partant de là, nous considérons que le conflit qui nous préoccupe revêt la forme d'un conflit d'usage et de voisinage (Caron et Torre, 2006 ; Guérin et al., 2005), à la fois humain et animal, né d'une proximité avec des acteurs aux intérêts et aux visions du territoire divergents et avec des animaux aux spatialités intrusives. Or, pour analyser les ressorts de tels conflits, les deux angles de vue de la sociologie et de la géographie gagnent à être articulés. D'un côté, la sociologie de l'action organisée se focalise sur les stratégies des acteurs visant à investir toute forme d'incertitude comme source de pouvoir (Friedberg, 1993). En particulier, le jeu et le placement des acteurs par rapport aux règles constituent des compétences sociales, source de pouvoir. De l'autre, c'est par l'angle spatial que la géographie étudie le jeu des acteurs. M. Lussault (2009) se penche en particulier sur la manière dont les acteurs cherchent la maîtrise spatiale, en usant de différentes compétences spatiales. En suivant son raisonnement, on peut alors considérer que le jeu des acteurs consiste à maîtriser et, donc, à rendre certain l'espace des autres acteurs ou actants. Par ailleurs, il définit les « opérateurs spatiaux » comme des entités qui possèdent une « capacité à agir avec performance dans l'espace géographique des sociétés concernées » (Lussault, 2007). Les non humains et les humains sont donc appréhendés en tant qu'opérateurs sociospatiaux, c'est-à-dire aux potentielles compétences à la fois sociales et spatiales.

Nous prenons donc les animaux sauvages « à problème» comme des révélateurs mais aussi comme des instigateurs des processus de mutation de ces territoires. Plus particulièrement, c'est au bouleversement provoqué par les sangliers dans les relations entre les acteurs que nous nous intéressons. L'objectif est de saisir la capacité du sanglier à imposer une réorganisation sociale et spatiale dans ces territoires : crée-t-il ou met-il à jour de nouveaux liens sociaux, de nouvelles spatialités, voire de nouvelles territorialités ${ }^{4}$ ? Autrement dit, s'agit-il d'un opérateur social, spatial, voire territorial? Dans ce cas, comment s'agencent les collectifs (Callon, 1986) d'hommes et d'animaux et de quelle manière les acteurs s'entendent-ils sur un vivre-ensemble commun ? Notre attention se porte sur la nature des liens socio-spatiaux reliant les acteurs et sur les modalités de leur agencement, c'està-dire sur les « artifices façonnés par les êtres humains pour équiper leur vie ensemble » (Thevenot, 2004). Or, la manière dont s'expriment localement les conflits est éminemment variable (Torre et al., 2006) : nous tenterons donc de mettre à jour les spécificités dans la manière dont les hommes et les animaux sont reliés.

Après une présentation des terrains prospectés et de la méthodologie adoptée, nous explorerons la manière dont s'agencent des modes de vie communs entre hommes et

4. Nous entendons par territorialité « une identité territoriale d'un individu ou d'un collectif » (Di Méo, 2003) ou, autrement dit, un territoire vécu et approprié (Debarbieux, 2003). 
Tableau 1. Caractéristiques des terrains étudiés

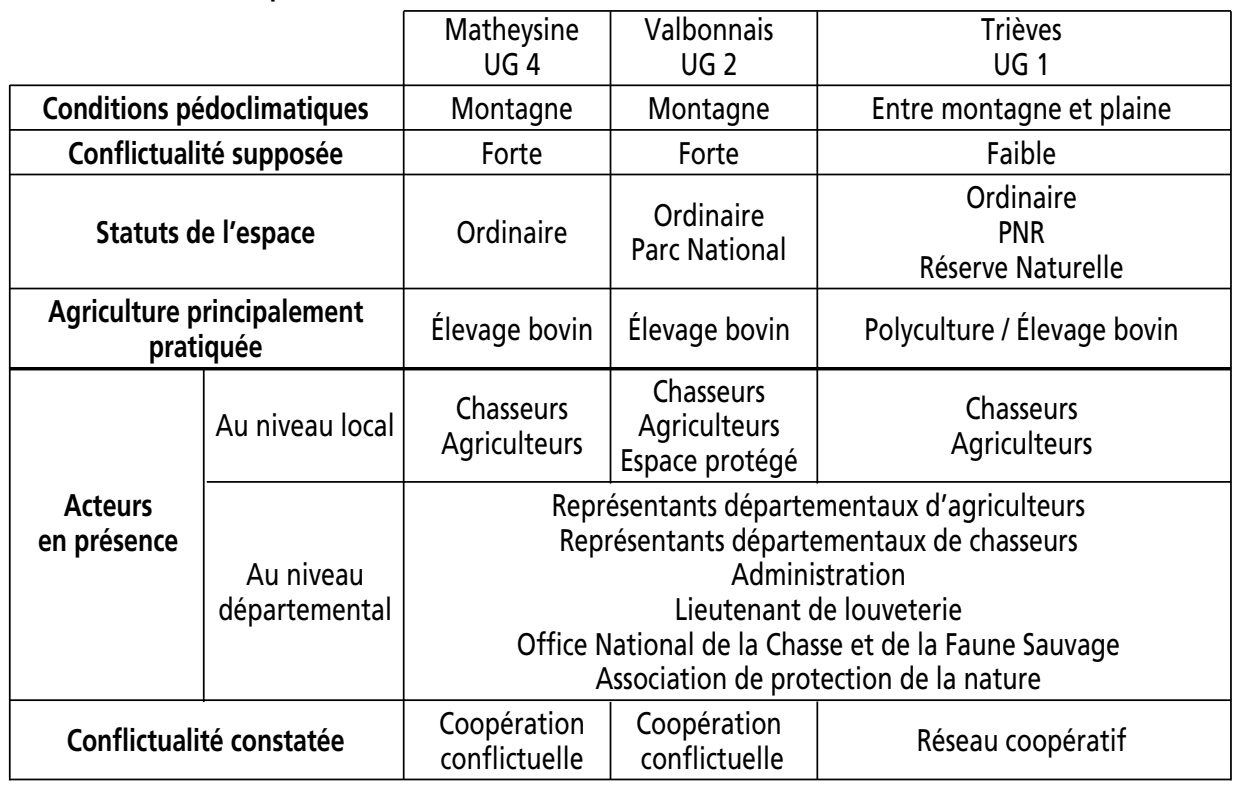

avec les animaux. Nous tenterons de comprendre dans un premier temps ce qui sous-tend les conflits autour du sanglier. L'expression spécifique de ces conflits et des arrangements locaux pour vivre ensemble sera mise en lumière dans un second temps : nous étudierons pour cela les comportements particuliers des animaux, les recompositions sociales puis spatiales qu'ils engendrent ainsi que les territorialités des acteurs qu'ils mettent en lumière.

\section{Les terrains étudiés et l'enquête}

Les résultats présentés se fondent sur la prospection, en 2005, de quatre terrains dans l'Isère. La chasse au sanglier y est basée sur un découpage multiscalaire du département. Les chasseurs pratiquent leur activité au niveau communal, au sein d'une Association communale de chasse agréée (ACCA), l'ensemble des ACCA étant regroupé au sein de la Fédération départementale des chasseurs de l'Isère (FDCI). Le territoire des sangliers étant généralement plus vaste que la superficie d'une commune, l'unité de gestion (UG) entend proposer une assise spatiale de la gestion du sanglier, adaptée à ses comportements spatiaux. Regroupant plusieurs ACCA, elle tente de respecter une certaine homogénéité géographique vis-à-vis du sanglier et est supposée réunir un même type de problématique cynégétique. À chacune de ces unités de gestion, est rattaché un comité local de gestion, composé des différents acteurs concernés (agriculteurs, chasseurs, forestiers, etc.) qui s'entendent sur un plan de gestion du sanglier, validé ensuite par les différentes instances des échelons supérieurs ${ }^{5}$.

Depuis les années 1990, avec la progression d'une chasse populaire du sanglier, la FDCI a mené une politique de développement de ces populations, à travers la promotion de pratiques cynégétiques conservatrices : les tableaux de chasse se sont enrichis, pour atteindre des records en 2000 , avec un peu plus de 5700 sangliers tués sur le département contre un peu plus de 3300 en 1995. Mais l'augmentation des populations de sanglier s'est traduite par

5. Le schéma départemental de gestion cynégétique n'était pas intégralement adopté en 2005. 
Tableau 2. Répartition des entretiens, selon le type d'acteur interrogé

\begin{tabular}{l|c}
\hline Types d'acteur ou de structure interrogés & $\begin{array}{c}\text { Nombre d'acteurs } \\
\text { interrogés }\end{array}$ \\
\hline Chasseurs & 12 \\
\hline Monde agricole & 12 \\
\hline Espaces protégés & 2 \\
\hline Association de protection de la nature & 1 \\
\hline Administration, Office National de la Chasse et de la Faune Sauvage, & 8 \\
\hline Lieutenant de louveterie & 35 \\
\hline Total & \\
\hline
\end{tabular}

une montée en puissance des dégâts sur les espaces cultivés et, par conséquent, des conflits opposant chasseurs et agriculteurs. En 2001, face à la recrudescence des plaintes d'agriculteurs adressées à leur intention, la Direction départementale de l'agriculture et de la forêt (DDAF) ${ }^{6}$ et le Préfet ont menacé la FDCI de déclasser le sanglier d'espèce gibier en espèce nuisible, si aucun accord n'était trouvé avec le monde agricole. Cette dernière a alors impulsé une politique de concertation dans l'ensemble du département et incité les représentants cynégétiques et agricoles de seize unités de gestion problématiques à formaliser des accords sur les modalités de gestion de l'animal, par des « conventions agro-cynégétiques ».

Une pré-enquête a permis de repérer ces unités problématiques et plus particulièrement les communes sujettes à conflit : la carte 1 indique quelques-unes de ces « communes a priori conflictuelles ». Parmi l'ensemble des unités concernées, les terrains d'études ont été ensuite sélectionnés selon leur situation dans des zones montagnardes ou non, leur degré de conflictualité supposée, ou encore le statut de l'espace. Nous évoquerons le cas des trois principales unités de gestion étudiées : le Valbonnais (UG 2), la Matheysine (UG 4) et le Trièves (UG 1) (tableau 1). Dans les deux premières, considérées par des acteurs clefs du département comme des « points noirs », l'effort de concertation s'est soldé par un

6. Aujourd'hui, Direction départementale des territoires (DDT). échec : le sanglier a été déclassé jusqu'à un accord ultérieur (respectivement, en 2004 et 2002). Les communes étudiées sont occupées par des exploitations agricoles principalement orientées sur de l'élevage bovin (lait et viande), fortement contraintes par un climat et un relief de montagne. En revanche, les relations entre agriculteurs et chasseurs sont plus apaisées dans le Trièves (un accord a été trouvé dès 2001). Bénéficiant de conditions pédoclimatiques plus favorables qu'en montagne, l'activité agricole y est plus diversifiée et plus dynamique. De nombreuses exploitations sont en polyculture-élevage, avec un élevage principal en bovin lait et viande. Précurseur dans des démarches d'agriculture durable et de qualité depuis plusieurs décennies, ce secteur est le plus dynamique de l'ensemble du territoire Apes Sud Isère. Enfin, le développement des populations de sangliers y est moins récent qu'en zone de montagne.

L'analyse porte sur trente cinq entretiens semi-directifs réalisés auprès des acteurs concernés par la gestion du sanglier, qu'ils soient impliqués localement dans les UG ou au niveau départemental : chasseurs et agriculteurs locaux, acteurs d'espaces protégés, administrateurs et techniciens de fédération des chasseurs, acteurs de l'administration ou encore d'association de protection de la nature (tableaux 1 et 2). Une première liste d'acteurs à interroger a été établie puis complétée au fur et à mesure de l'avancée des entretiens, par le croisement des dires des acteurs, pour déterminer les acteurs « pertinents » (Friedberg, 1993). La 

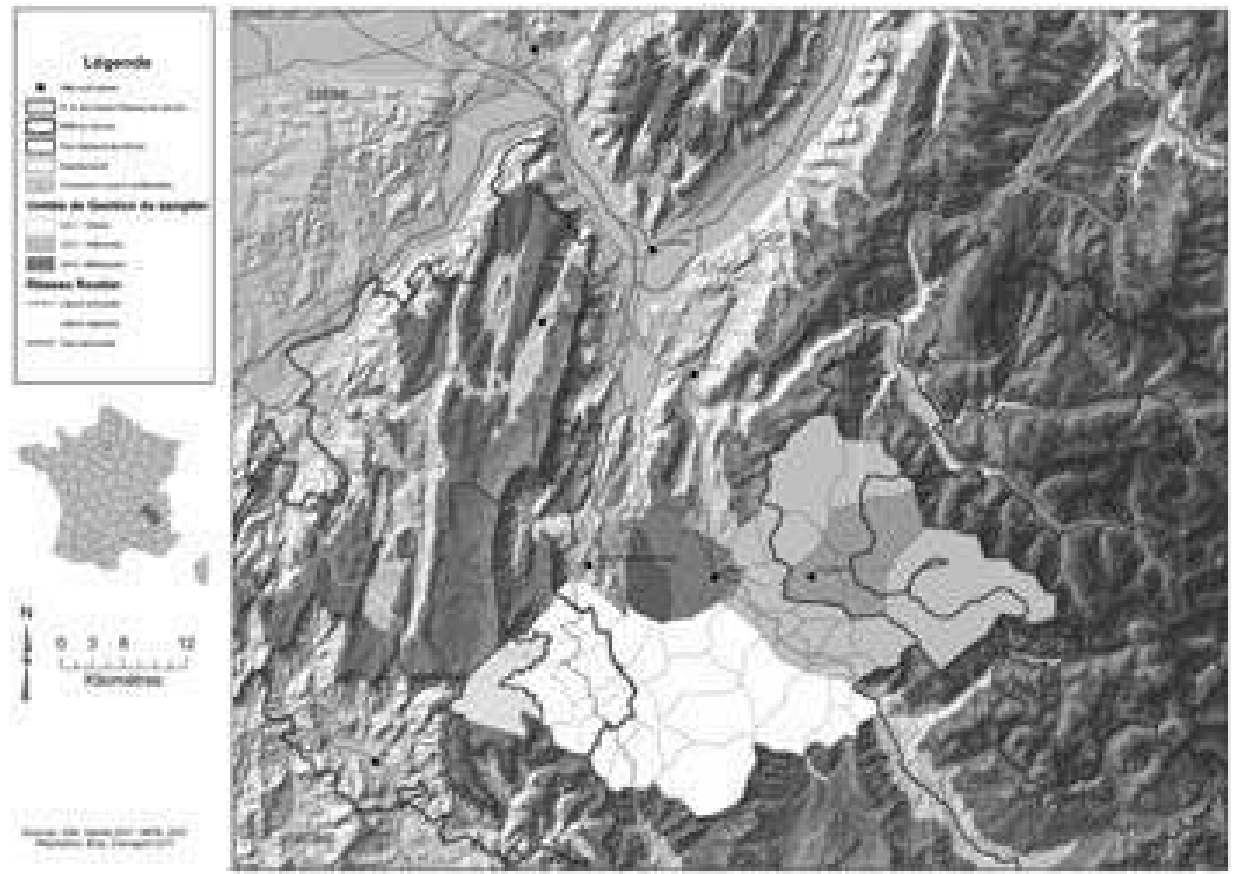

construction d'un échantillon qualitatif a donc été progressive et s'est fondée sur une double sélection des acteurs : ceux-ci ont été choisis selon leur appartenance à un groupe social concerné par le conflit ainsi qu'à une " scène locale » étudiée (Decrop et al., 2001). Si le choix des acteurs est qualitatif, c'est, à l'instar de la «saturation des modèles » (Kaufmann, 1996), la saturation des arguments qui a déterminé l'arrêt des entretiens.

Pour dégager les expressions du conflit communes à l'ensemble des territoires, le corpus des entretiens a été complété et comparé à d'autres sources documentaires telles que des articles de presse régionale ( $L e$ Dauphiné Libéré) et d'une revue cynégétique (Sanglier passion). Enfin, pour dégager les formes d'expression spécifique du conflit au niveau local, le corpus a été enrichi par l'étude de documents décrivant une formalisation locale ou émis par des parties prenantes.

Le dépouillement des entretiens s'est basé sur deux méthodes d'analyse : l'analyse entretien par entretien et l'analyse thématique. La grille utilisée reprend les thématiques du guide d'entretien mais a également été complétée au fur et à mesure de l'analyse, avec l'émergence de nouveaux thèmes.

\section{Des divergences révélées par le sanglier}

Passant de l'image de la chasse cueillette à celle d'une " chasse gestion » visant un équilibre agro-sylvo-cynégétique (Traïni, 2004), l'activité cynégétique tend aujourd'hui à être présentée par ses tenants comme une " chasse écologique », voire durable (Ginelli, 2008 ; Ginelli et Le Floch, 2006). Telle qu'elle est organisée, la chasse au sanglier semble en effet répondre aux préceptes de la gestion durable des animaux. D'un point de vue environnemental, elle s'est dotée d'outils pour gérer les populations de sangliers, grâce à des indicateurs établis à partir des tableaux de chasse et des montants financiers des indemnisations de 
dégâts. Au niveau économique, les retombées négatives des populations de sangliers sont compensées et des dispositifs de protection sont installés autour des cultures pour les réduire au minimum. Enfin, au niveau social, la gestion fait l'objet de concertation et d'entente entre représentants des acteurs concernés, au sein de l'unité de gestion. Pourtant, la prise en compte des trois pôles d'une gestion durable ne suffit pas à réduire les désaccords et les conflits. Au contraire, elle les cristallise, en alimentant les controverses qui en sont à l'origine : les acteurs ne s'accordent pas sur ce que doit être une gestion durable, aux plans environnemental, économique et social.

\section{Des controverses...}

L'étude du discours des acteurs met à jour des arguments génériques opposés qui alimentent ces controverses et structurent le conflit par une opposition entre partisans et détracteurs des sangliers. Le conflit montre en effet des réagencements sociaux transversaux aux groupes sociaux stables : il oppose les suscrophobes ${ }^{7}$, essentiellement composés d'acteurs du monde agricole et rejoints par certains chasseurs non-chasseurs de sanglier, aux suscrophiles, rassemblant généralement des chasseurs de sanglier. Chaque camp possède une gamme d'argumentation pour asseoir sa posture, que l'on peut lire au travers des trois pôles de la durabilité.

Au plan économique, les suscrophobes et les suscrophiles n'interprètent pas de la même manière les dégâts de sangliers. Pour les premiers, l'ongulé entraîne de fortes pertes économiques, que les indemnisations ne couvrent pas toujours. À l'inverse, pour les seconds, les dégâts ne seraient pas si importants que les agriculteurs veulent bien le dire et les pertes de céréales seraient pratiquement anecdotiques en comparaison de la production départementale.

7. En référence au nom latin du sanglier, Sus Scrofa.
Mais les diverses controverses d'ordre économique s'entremêlent à des controverses portant sur l'aspect social de la gestion du sanglier. Selon les suscrophiles, l'impact économique du sanglier serait exagéré car instrumentalisé par une profession agricole en proie à d'importantes difficultés structurelles. L'animal servirait de bouc émissaire et les syndicats agricoles useraient de cet artifice pour détourner l'attention des réels problèmes sociaux et économiques plus profonds de la profession. Les suscrophobes, quant à eux, dénoncent le pouvoir d'expertise et d'action laissé aux chasseurs dans la gestion du sanglier. Jugeant que les populations sont trop basses, les chasseurs décréteraient la fin de la chasse avant l'heure et favoriseraient ainsi leur développement. De même, pouvant agir à leur guise malgré les accords issus du comité local de gestion, ils ne tueraient pas assez de sangliers et se justifieraient par la difficulté de la chasse. Enfin, la manière dont les chasseurs peuvent s'approprier l'espace dans leur activité cynégétique ne les satisfait pas : il leur semble que leur droit de propriété est parfois oublié, voire bafoué par le droit d'usage, qu'ils ne contestent pourtant pas aux chasseurs. Derrière de tels arguments, les agriculteurs montrent leur désapprobation de la mutation sociale actuelle des territoires ruraux, avec l'abandon des valeurs de travail dont ils se pensent les porteurs, au profit des activités récréatives. Si les paysans $^{8}$ regrettent leur perte progressive de légitimité économique et sociale au sein des territoires ruraux, les chasseurs, quant à eux, justifient leur activité de chasse à travers un argumentaire à la fois environnemental et entrepreneurial (Dalla Bernardina, 1996) ou industriel (Traïni, op. cit., en référence à Boltanski et Thévenot, 1991). Ils estiment que les effectifs de sangliers ont augmenté mais ne les considèrent pas

8. Certains de nos interlocuteurs se sont désignés par ce terme lors des entretiens. 
en surpopulation. Au contraire, la gestion cynégétique qu'ils mènent viserait à atteindre un équilibre agro-sylvo-cynégétique. Pour cela, ils s'appliqueraient à prélever des animaux, mais de manière raisonnable, pour préserver le « capital » de sangliers. À l'inverse, sur ce registre, les suscrophobes délégitiment la pratique de la chasse comme outil de gestion environnementale. Selon eux, loin de réguler correctement la faune sauvage, les chasseurs seraient à l'origine d'une surpopulation d'animaux non sauvages. C'est notamment par des lâchers de cochongliers qu'ils seraient parvenus à abâtardir les populations de sangliers existantes. En outre, sous prétexte d'utiliser un outil de dissuasion, les chasseurs useraient de l'agrainage ${ }^{9}$ pour nourrir et fidéliser les compagnies dans leur commune. Prenant le " goût du maïs $»^{10}$, les ongulés perdraient non seulement leur caractère sauvage mais seraient en plus définitivement conditionnés à cet aliment très appétant, qu'ils rechercheraient dans les champs cultivés.

\section{2. ...et des postures génériques}

Les acteurs impliqués dans ces controverses sont donc regroupés de manière innovante, en regard des catégories d'acteurs définies a priori. Le conflit a en effet engendré une redéfinition des contours des groupes d'acteurs en présence (Bossuet, 2007). Mais la récurrence de ces regroupements sociaux nous a amené à les considérer comme des innovations stabilisées, représentant en quelque sorte une nouvelle « pré-structuration » (Friedberg, op. cit.) des conflits : les postures qualifiées de

9. Distribution de maïs ou de céréale appétante en vue d'attirer les sangliers dans un secteur géographique, pour les détourner des cultures.

10. À l'instar des chiens errants, qui, prenant le «goût du sang », sont définitivement perdus comme animaux domestiques (Bobbé, 1999), l'ingestion de céréales domestiques chez des sangliers sauvages leur ferait franchir la frontière sauvage/domestique et les désensauvagerait. «macrosociales » constituent pour chaque acteur une affirmation de l'appartenance à un des camps en présence et relèvent d'une volonté d'exclusion des arguments de l'autre, de fermeture du débat (Hirschman, 1991). Mais si les acteurs portent de tels discours incompatibles, ils développent également des postures à l'origine d'arrangements locaux. D'autres logiques, intrinsèquement liées à la spécificité de chaque territoire et de ses opérateurs, sont à l'œuvre dans la composition du vivreensemble.

\section{Expression spécifique des conflits et arrangements locaux}

\section{Des principes communs aux postures microsociales}

Construites dans la confrontation quotidienne aux tenants du camp opposé, liées à la proximité géographique et à l'individualisation des protagonistes, les « logiques microsociales » prennent le relais des postures stéréotypées et, par leur caractère généralement consensuel, permettent des arrangements locaux. Si elles s'expriment dans des vivre-ensemble spécifiques, elles s'appuient sur des principes communs. C'est par exemple le cas lorsque des principes supérieurs communs de bon voisinage, liés à la proximité des acteurs et la quotidienneté de leurs relations, prennent le relais des postures stéréotypées. Les acteurs n'agissent pas alors en tant que suscrophiles ou suscrophobes mais en tant que bons voisins, se rendant mutuellement des services.

Ainsi là où le vivre-ensemble est apaisé, certains chasseurs viennent prêter mainforte aux agriculteurs lors des grands travaux tels que la récolte du foin ou encore participent à la remise en état des champs dévastés par les sangliers. Au travers de tels services rendus, les chasseurs apportent une reconnaissance des désagréments subis par les agriculteurs et, plus largement, de leur légitimité sociale et économique au 
sein du territoire. Le rapport entre agriculteurs et chasseurs est dans ce cas gouverné parce que nous avons appelé des « rationalités affectives » (Mounet, 2007). La rationalité affective reprend la notion d'action affective définie par Weber, en lui attribuant une dimension plus réfléchie. Créatrice d'un « microsocial » apaisé, elle répond plutôt à une éthique de responsabilité (Weber, 1971).

Mais si l'individualisation des protagonistes, liée à la proximité peut être synonyme de potentiel apaisement, elle peut être, à l'inverse, à l'origine de forts conflits (Caron et Torre, 2006). Des inimitiés entre individus peuvent donner lieu à des logiques microsociales de type conflictuel. Certains acteurs expliquent ainsi des blocages au sein de la Matheysine par des problèmes de personnes. Le sanglier serait un prétexte pour exprimer des conflits d'une autre teneur, faits de rancœurs entre individus. Derrière des arguments génériques, il y aurait donc des règlements de compte invisibles pour qui ne connaît pas l'histoire des relations entre les individus.

«Tu connais [...] Manon des sources. [...] Tu as vraiment l'impression de revivre ça par moments sur le terrain. [...] Quand on te présente un problème, ben, essaye de voir par-derrière tout ce qu'il y a. Et tu découvres des règlements de comptes entre les uns et les autres. [...]. Et la chasse, c'est ça, c'est vraiment des petites histoires. »

(Un acteur administratif)

En outre, l'individualisation des protagonistes peut également aboutir à une régulation des conflits par la peur, basée sur des rapports de force peu équitables. C'est le cas lorsque des chasseurs menacent de représailles des agriculteurs protestant auprès des administrations contre les dégâts de sangliers. La violence fortement présente dans la chasse au sanglier (Vourc'h et Pelosse, 1988) est redoutée par les acteurs locaux.

«Un truc qu'on peut pas écrire mais, avec le sanglier, il y a du terrorisme intellectuel. T'as des gars, c'est des violents, certains. [...] Avec l'agriculteur local, ils vont lui faire comprendre que bon, [...], ils vont faire des efforts et tout et puis si le gars, il a envie de faire de son côté... ben, ils vont le menacer, quoi. [...] C'est pas quantifiable. Une grange qui brûle... Une voiture qui... Non, non mais je veux dire il y a des violents parmi les chasseurs de sangliers, il y a des violents. [...] Par derrière, il s'en passe, hein.»

(Un acteur administratif)

Les arrangements locaux et le niveau de conflictualité des territoires dépendent notamment de l'équilibre entre postures macrosociales et microsociales. Or, le jeu des acteurs qui gouverne cet équilibre dépend de nombreux éléments :

- la présence et le rôle actif ou non des différents opérateurs humains et nonhumains (l'implication d'opérateurs tiers dans le conflit chasseur/agriculteur peut modifier les postures et les stratégies des uns et des autres) ;

- l'histoire du territoire (comme le dynamisme agricole dans le cas du Trièves), l'histoire des relations entre acteurs ; - les caractéristiques individuelles des hommes ou des animaux mais aussi la variabilité des engagements et des stratégies des acteurs.

Nous aborderons quelques-uns de ces éléments pour saisir en quoi les sangliers peuvent impulser des liens spécifiques sociospatiaux sur les terrains étudiés.

\section{Des comportements particuliers chez les animaux}

Tout d'abord, le comportement des sangliers intervient sur les accords potentiels entre acteurs. Selon leur spatialité et ce qu'ils apprennent des comportements humains, ces animaux peuvent en effet entraver les éventuels compromis.

C'est le cas lorsque les sangliers montrent une affection particulière pour des secteurs, caractérisés par exemple par un climat plus clément ou une absence d'intervention cyné- 
gétique induisant un «effet réserve ${ }^{11}$, c'est-à-dire le cantonnement des populations de sangliers dans des secteurs non chassés. Dans ce dernier cas, lorsque la concentration de sangliers est particulièrement importante, les individus peuvent alors perpétrer des dégâts en périphérie de la réserve.

C'est également le cas lorsque des individus apprennent à passer outre les dispositifs de protection mis en place par les chasseurs et les agriculteurs. Des animaux s'habituent ainsi aux dispositifs d'effarouchement ou aux clôtures électriques défendant les cultures.

"Et puis on a mis un effaroucheur avec un système aléatoire qui alimente l'autoradio. Ca marchait au début. Après, ils s'habituent vite. Le fils du cousin de Joseph, il a trouvé des cochons couchés sous les hauts parleurs!...»

(Un chasseur)

De même, Dedes individus semblent se spécialiser sur une alimentation d'origine agricole. C'est le cas de sangliers qui traversent régulièrement les clôtures électriques pour manger les maiis en lait (stade de maturité du mais) et vont même jusqu'à enfreindre le domus, en se servant dans les silos de maïs.

« À partir du moment où l'animal vient chez toi pour faire du dégât, à l'intérieur de tes bâtiments, sincèrement, ça te fout les boules, ça te fout les boules, ça te fout les boules... »

(Un agriculteur)

Outre les dispositifs de protection fixes, des sangliers parviennent également à déjouer les interventions humaines visant à leur faire respecter les frontières ager/silva. Ils apprennent ainsi à échapper aux tirs des lieutenants de louveterie mandatés pour effectuer des prélèvements nocturnes d'animaux en cas de dégâts importants.

Ces exemples montrent en quoi les attitudes des sangliers peuvent influer sur la

11. Décrit dans de nombreux écrits de l'ONCFS et de l'INRA. Par exemple, Tolon et al., 2007. possibilité d'une entente entre humains. Ils permettent également de revenir sur l'angle d'analyse adopté par Poinsot (2008) pour aborder les questions de recoupement des territoires cynégétiques communaux et des spatialités animales dont les métriques diffèrent. En particulier, Poinsot et Saldaqui (2009) s'intéressent aux éléments influençant les arrangements locaux, nécessaires à la mise en place d'une gestion supra communale cohérente. Or, la posture théorique que nous adoptons, en considérant les animaux comme des opérateurs potentiels, permet de saisir ce recoupement sous un angle particulier et de mettre à jour d'autres éléments influençant l'expression spécifique des vivreensemble, comme la capacité d'apprentissage des animaux à une échelle collective (effet réserve) mais aussi et surtout à une échelle individuelle.

Étudions à présent l'influence du jeu des acteurs sur l'expression spécifique des conflits et, finalement, le type de vivreensemble au sein du territoire.

\section{Recomposition sociale et stratégies des acteurs}

Nous l'avons évoqué, les relations entre les acteurs peuvent être analysées par la question du pouvoir (Friedberg, op. cit.). «L'équilibre stratégique » obtenu dans les contextes d'action est le résultat d'un bricolage de la part des acteurs au sein d'une coopération conflictuelle, qu'elle soit plus ou moins conflictuelle ou coopérative ; il peut également être le résultat d'un accord orienté vers un principe supérieur commun (Boltanski et Thévenot, op. cit.) au sein d'un réseau de type coopératif (Callon, $o p$. cit.) Dans les deux cas, l'équilibre est instable et contingent et peut être renégocié à tout instant selon les acteurs et les actants non humains ou les opérateurs présents et actifs dans le territoire. Cette variabilité ne permet donc pas de définir un état de conflictualité stable pour chaque territoire étudié car, de manière périodique, 
les coopérations conflictuelles passent par des équilibres plus ou moins conflictuels ou apaisés ( $c$ f. tableau 1 ).

Les trois types de logiques macrosociales et microsociales présentées précédemment sous-tendent la gamme de jeu utilisée par les acteurs, mobilisée et dosée différemment selon l'équilibre stratégique ou le vivreensemble dans lequel sont pris les acteurs. En effet, ce sont principalement les relations interpersonnelles, le microsocial, qui régissent les actions dans le réseau qui caractérise le Trièves. Quant aux coopérations conflictuelles (Matheysine et Valbonnais), leurs acteurs montrent une gamme de jeu plus diversifiée, agissant tantôt en acteur macrosocial, tantôt en individu microsocial. Le vivre-ensemble avec des «partenaires-adversaires » (Friedberg, op. cit.) complexifie le jeu : à trop user de macrosocial, les acteurs risquent de provoquer une rupture de dialogue définitive et de basculer leur territoire dans un conflit permanent ; à trop mobiliser de microsocial coopératif, ils risquent de perdre leur pouvoir de contestation. L'ouverture aux autres contextes d'action, locaux ou à l'échelle macro, n'a également pas la même signification selon que les acteurs sont pris dans un réseau coopératif ou une coopération conflictuelle. Alors que, dans le premier cas, elle a pour objectif de conforter le bon vivre-ensemble, elle est plutôt utilisée dans le second cas dans le but d'orienter le vivre-ensemble favorablement à l'acteur.

\section{L'ouverture aux échelles supérieures ${ }^{12}$}

Ainsi, selon le vivre-ensemble, la mobilisation du cadre macro, c'est-à-dire le niveau départemental n'est pas utilisée et perçue de

12. Précisons que si les logiques microsociales ont été étudiées au niveau local, elles n'en sont pas l'apanage. De plus, les acteurs montrent une variabilité d'engagement (Thévenot, 2006) selon les scènes dans lesquels ils sont pris. En particulier, les acteurs « multicasquette» (Gumuchian et al., 2003) peuvent adopter des postures différentes selon le rôle et influencer les vivre-ensemble locaux. la même façon par les acteurs. Elle peut se traduire par une intervention de lieutenant de louveterie, généralement très mal perçue par les chasseurs lorsqu'elle leur est imposée. Dans le cadre des coopérations conflictuelles, elle est donc généralement synonyme de stratégie offensive des acteurs pour reprendre le pouvoir dans le rapport de force qui les oppose à leurs protagonistes. Par exemple, dans la Matheysine, plutôt que de privilégier l'entente locale, cet agriculteur s'adresse directement à la DDAF - aujourd'hui, DDT -dès qu'un problème survient.

« Normalement, il faut déjà qu'on voye avec les chasseurs s'ils sont d'accord. Comme les $3 / 4 d u$ temps, ça marche pas, on va directement à la DDA chez [l'agent chargé du dossier]. »

(Un agriculteur)

Ne voulant pas négocier avec le représentant de l'unité de gestion, il préfère mobiliser les échelons supérieurs. Par ce biais, il remet non seulement en question le protocole officiel et la légitimité de ce représentant des chasseurs mais il montre, en plus, une capacité d'autonomie qui lui confère du pouvoir dans le contexte d'action. Ce chasseur interprète une telle attitude interdisant tout compromis comme une action refusant toute dimension « affective » des rapports sociaux.

"Oui, disons que les agriculteurs. Bon, eux, c'est ... je sais pas si vous connaissez bien leur milieu mais c'est... c'est dur, hein, comme milieu. Alors, ils ne font pas de sentiment. Ils passent direct par en haut et puis ils ont les portes ouvertes partout. »

(Un chasseur)

Cette stratégie offensive déclenche alors des conflits ou, tout du moins, des représailles auprès des agriculteurs qui en sont à l'origine.
"Le lendemain matin, toutes les clôtures de mes blés, tout arraché, tout balancé. J'avais les postes de clôture avec les panneaux solaires, tout balancé, tout massacré. »
(Un agriculteur)

À l'inverse, dans le réseau coopératif du Trièves, la régulation des tensions se fait au sein même du territoire et n'aboutit que très 
rarement à un recours aux échelles supérieures. C'est plutôt vers l'administrateur de la fédération dont la pertinence en tant que médiateur est acceptée par tous que les acteurs se tournent. Ainsi, cet agriculteur confronté à un sanglier à l'origine d'importants dégâts se refuse, malgré tout, à faire appel à un lieutenant de louveterie. À nouveau, le passage de l'entretien que nous avons souligné montre bien la rationalité affective à l'œuvre dans les justifications de cet acteur.

«Non, pour le sanglier, non. Attends, moi je vois pas... ça, ça fait partie du politique. Je vais appeler [l'administrateur] pour dire tu déclenches une opération avec les lieutenants de louveterie? Attends, l'ACCA, ils comprennent plus. Ils me disent "t'es un salaud". Moi, je suis plutôt... si tu veux, c'est facile de dire on l'a raté, on l'a pas vu... ils [les chasseurs] vont pas dire "on veut pas le tuer ". Un jour, il crèvera et puis voilà. Tant que c'est du vivable... Si tu veux, ça nous fait chi... de ressemer nos $3000 \mathrm{~m}^{2}$ mais c'est que du compromis, hein... partout dans la vie. »

(Un agriculteur)

Bien que ces agriculteurs se plaignent des chasseurs et usent d'arguments génériques à leur égard, les discours tenus face à l'administration donnent donc l'image d'un bon « vivre ensemble ».

\section{Une perméabilité aux contextes d'action de même échelle}

Comme dans le cas de l'ouverture aux échelles supérieures, les contextes d'action locaux sont instrumentalisés par les acteurs dans les coopérations conflictuelles. C'est le cas, par exemple, des chasseurs qui, en réponse à une stratégie offensive d'un agriculteur, dénoncent des pratiques agricoles non conformes à la législation auprès des instances départementales.

"En plus, un autre truc. On a une vache qui crève, elle s'était dérochée. On pouvait pas la sortir avec un tracteur, la seule solution, c'était de la sortir avec l'hélicoptère et ça coûtait 5000 francs. Alors, on dit, bon, ben, allez, on l'enterre. Normalement, il faut demander des autorisations, c'était un samedi, on a dit on l'enterre. Et puis, [...] là-haut, on a des alpages où y a des dégâts de sangliers. On dit on va faire une déclaration pour dire qu'il y a trop de dégâts. Y et toute son équipe : ah, il veut nous embêter? Ils ont fait une lettre à la DSV $V^{13}$ comme quoi on avait enterré une vache. [Comme ça a été] laissé sans suite ils sont allés voir [le technicien du Conseil Général], [il] n'a pas voulu marcher. Ils sont allés voir FR3, ils ne sont pas montés. Mais c'est pour vous dire au point où c'en est! »

(Un agriculteur)

Ici, la stratégie défensive des chasseurs n'est pas développée dans le contexte d'action autour du problème du sanglier mais dans un contexte d'action apparemment déconnecté de ce conflit.

À l'inverse, l'ouverture aux contextes d'action locaux dans le Trièves revêt une signification bien différente. C'est le cas d'un agriculteur siégeant au comité de gestion qui utilise son statut pour alimenter les relations vertueuses avec les chasseurs de sa commune. Fonctionnant sur du donnantdonnant, il négocie implicitement des tirs plus nombreux sur les sangliers contre une pression auprès de la fédération pour obtenir des bracelets de chevreuil.

« [J'ai aidé les chasseurs] en faisant une lettre à la fédé [fédération des chasseurs de l'Isère], en disant qu'il y a beaucoup de dégâts de chevreuil, que la population est très importante et que pour nous, il faut qu'il y ait plus de chevreuils à abattre. (rires) Tu vois... Donc, ça, ça fait partie, c'est du politique, ça. Ça fait partie du donnant-donnant. Ça veut dire, je te laisse ça mais cet automne, le sanglier, vous irez un peu plus. Donc, nous, ça se passe pas mal. »

(Un agriculteur)

Ces deux exemples montrent en quoi la perméabilité aux autres contextes d'action peut être utilisée différemment par les acteurs, selon la forme du vivre-ensemble dans lequel ils sont pris.

Mais de manière plus générale, cette perméabilité nous amène à nous interroger sur la définition du conflit que donnent des auteurs comme Mélé (2003) ou Torre et al. (2005). Selon le premier, la notion de conflit doit être attribuée à des situations «qui

13. Direction des services vétérinaires. 
constituent une manifestation de protestation ou d'opposition » explicites et publiques; le second considère qu'il est nécessaire de distinguer conflit et tension, à travers « l'engagement ».

Or, si l'on applique un tel raisonnement aux territoires étudiés et que l'on s'attache à analyser les seuls engagements d'acteurs sur le dossier "sanglier», la vision adoptée est partielle. Toutes les formes d'engagement, découlant de stratégies offensives ou défensives des acteurs mais prenant pied dans d'autres contextes d'action seraient en effet occultées. De même, le seul indicateur de l'engagement dans le cas de l'exemple pris sur le Trièves aboutirait à de fausses conclusions, celle d'un conflit autour du chevreuil. Les conflits autour du sanglier nous amènent ainsi à remettre en question le seul indicateur de l'engagement officiel pour différencier la tension ou le conflit latent du conflit. Il est nécessaire d'être attentif à l'ensemble des engagements explicites ou plus implicites mais également s'exprimant dans l'ensemble des contextes d'action concernés par les acteurs en conflit.

\section{Du lien spatial, des enjeux spatiaux, de nouvelles territorialités?}

Outre les recompositions sociales qu'ils engendrent, les sangliers provoquent également des bouleversements dans les liens spatiaux qui lient les acteurs les uns aux autres.

Tout d'abord, les stratégies sociales mises en œuvre par les acteurs montrent une dimension spatiale. Les formes de coopération entre les acteurs s'inscrivent dans l'espace : la mise en place de dispositifs de protection tels que les effaroucheurs, les radios, les clôtures électriques marque la frontière spatiale entre les domaines du sauvage et du domestique. Ces marques spatiales peuvent devenir des enjeux de pouvoir sur lesquels les acteurs interviennent dans des phases conflictuelles. C'est le cas, comme nous l'avons évoqué précédemment, des chasseurs qui ont arraché les clôtures électriques d'un agriculteur ayant fait appel à l'administration pour régler des problèmes de dégâts. Le sanglier provoque donc des comportements spatiaux particuliers autour de nouveaux lieux ou objets spatiaux à enjeux.

Ensuite, les jeux des acteurs sur l'espace peuvent avoir une incidence sur la possibilité d'un accord social. Par exemple, les relations au sein du monde de la chasse jouent sur les compromis passés avec les agriculteurs. Sur une commune étudiée de la Matheysine où les conflits entre chasseurs sont particulièrement aigus, l'espace chassable est divisé en quatre à cinq équipes. Or, la multitude d'équipes induit de fortes rivalités : chacun veut s'accaparer les sangliers et éviter que les sangliers levés par ses soins ne se fassent tuer par l'équipe adverse.

"Le problème qui était récurrent sur la commune $X$, c'est un problème d'équipes de chasseurs. Donc vous en arrivez à ce que les chasseurs veulent pas lâcher leurs chiens pour pas que le sanglier se fasse tuer par l'équipe d'à côté. Euh... bon, le sanglier, il en profite. C'est ça, hein. »

(Un lieutenant de louveterie)

La concurrence entre équipes d'une même commune ou de communes différentes peut s'exprimer par des violences, parfois importantes, allant de la simple bagarre à l'incendie de grange ou l'abattage de chiens de chasse. Une telle ampleur des conflits est notamment expliquée par des chasseurs par la course au record qui gagne certains d'entre eux : l'enjeu est d'être celui qui possède le congélateur le plus fourni en fin de saison. Or, ces conflits au sein du monde de la chasse ont de fortes incidences sur le vivre-ensemble : lorsque des chasseurs d'une équipe tentent d'impulser un compromis avec le monde agricole, les chasseurs des équipes rivales peuvent refuser de collaborer.

Mais, enfin, si le sanglier crée de nouveaux liens spatiaux, est-il à l'origine de nouvelles territorialités ou, tout du moins, que nous apprend-il des territorialités des acteurs ? Nous l'avons évoqué, les unités de 
gestion lient des communes par des principes de gestion communs mais chaque chasseur pratique son activité cynégétique dans sa commune. Or, l'absence d'homogénéité de la répartition des sangliers peut interroger les liens entre communes (Poinsot et Saldaqui, op. cit.) et provoquer une concurrence, dégénérant en conflit. Par exemple, les chasseurs de communes particulièrement fournies en sangliers comme dans le cas de la Matheysine ont dû faire face au mécontentement des agriculteurs et augmenter leur effort de chasse pour limiter les dégâts. Pour cela, ils se sont engagés à pratiquer la « chasse à la neige », c'est-à-dire lorsque le sol est recouvert de neige. $\mathrm{La}$ plupart du temps, les ACCA décident de ne pas adopter cette pratique de chasse, de peur de prélever trop d'animaux : tuer des sangliers dans ces conditions est en effet beaucoup plus aisé. Les chasseurs des communes avoisinantes de la même UG, ayant pour leur part décrété la fin de la chasse au sanglier, ont alors eu le sentiment de se faire voler les sangliers qu'ils avaient voulu préserver par la fermeture de la chasse. Cet exemple montre en quoi le territoire de gestion du sanglier, l'unité de gestion, ne correspond pas réellement à la territorialité des acteurs, au territoire pour lequel ils entretiennent un sentiment d'appartenance.

Ce même phénomène est mis en évidence dans le Valbonnais où l'échec des tentatives de concertation en 2001 a entraîné le déclassement du sanglier. La réaction des chasseurs dans l'ensemble des communes ne se fit pas attendre : de nouveau, c'est en supprimant tout dispositif de protection qu'ils montrèrent leur désaccord. Or, dans les communes où chasseurs et agriculteurs s'entendaient relativement bien, cette nouvelle situation est venue perturber la « coopération conflictuelle » installée. Les agriculteurs, habitués à un climat plus coopératif dans leurs communes, ont contesté les mesures prises par le préfet et ont fait pression auprès de leurs représentants agricoles à l'origine du blocage et du déclassement juridique du sanglier. Une telle posture microsociale, mise en lumière par le changement du cadre juridique, a donc partagé le monde agricole, certains d'entre eux s'associant temporairement aux chasseurs pour obtenir un meilleur vivre-ensemble. Cet agriculteur, à l'origine du classement nuisible, fut stigmatisé par ses pairs.

"Déjà l'unité de gestion, on a eu des problèmes,
le Beaumont nous a reproché... des paysans qui
étaient contre, quand ça a été classé nuisible, ils
voulaient pas qu'on soit en nuisible parce que
sur le secteur, eux, depuis longtemps, on savait
même pas qu'il y avait des chasseurs qui par-
quaient leurs céréales. Et le jour où ça a été nui-
sible, ils ont rien trouvé de mieux que d'aller
démonter les parcs. » (Un agriculteur)

"Oui. Parce que l'ordre de la fédération a été donné, "ben ils ont pas signé, lundi tu ramènes tout le matériel chez moi ". Un administrateur qui dit ça. Ça, c'est pas très intelligent mais il l'a dit. » (Un agriculteur)

L'homogénéité des unités de gestion, est donc ici remise en question par des acteurs dont les territorialités diffèrent de cette maille administrative. Si la non-concordance des territoires vécus et appropriés avec les territoires politiques est fréquente, ce qui est intéressant dans ce cas précis, c'est que la disjonction entre de telles formes de territoire débouche sur des scissions au sein même du camp suscrophobe. Les divergences des territorialités et des territoires politiques mettent ainsi en lumière une tension entre postures macrosociale et microsociale, adoptées par les agriculteurs.

De même, le sanglier met à jour des sentiments de territorialités spoliées et montre la difficile articulation entre territoires de gestion, territoires de protection et territorialités dans l'unité de gestion du Valbonnais dont le périmètre est recoupé par le Parc national des Écrins. Les chasseurs accusent le parc d'être à l'origine d'un « effet réserve » et de protéger à tort des sangliers se rendant fautifs de dégâts. Par une telle accusation, les chasseurs sont parvenus à 
opérer un basculement du conflit, rangeant de leur côté et contre le Parc, les agriculteurs initialement accusateurs des résultats de gestion des chasseurs. La présence d'un opérateur tiers, le Parc des Écrins, a donc permis une association particulière, celle des agriculteurs et des chasseurs contre l'espace protégé. Les acteurs locaux, indépendamment de leur appartenance macrosociale, se liguent contre des instances de protection, porteuses, à l'origine, des valeurs nationales de protection et ressenties comme imposées au tissu social local. Ces postures microsociales reflètent ici la spécificité de ce territoire : le sanglier met à jour cette opposition latente entre identité locale et intérêts nationaux ${ }^{14}$.

S'il ne crée pas de nouvelles territorialités, le sanglier, par ses spatialités, met donc en lumière des problèmes de correspondances entre territoires de gestion et territorialités.

\section{Conclusion}

L'étude des expressions génériques et spécifiques du conflit nous amène à comprendre qu'au-delà des postures générales et stéréotypées, il existe des logiques spécifiques, d'ordre microsocial, débouchant sur des arrangements locaux particuliers à chaque territoire. En outre, au travers des stratégies et des engagements des acteurs dans leur territoire, on s'aperçoit que le sanglier provoque des réagencements sociaux, des nouveaux comportements spatiaux et met également à jour des distorsions entre territoire politique de gestion et territoires vécus et appropriés. À la lecture de ce social et de ce spatial « en train de se faire » (Latour, 2006), deux leçons peuvent être retenues pour une gestion durable du sanglier.

- Tout d'abord, la déconstruction des postures des acteurs met en évidence à

14. La modernisation de la loi de 1960 en 2006, avec l'obligation d'élaboration d'une charte en collaboration avec les acteurs locaux du territoire, peut avoir engendré une évolution des mentalités. quel point les aspects environnementaux, économiques et sociaux peuvent être entremêlés. Une gestion durable de la faune sauvage ne peut donc se penser sans prendre en compte les trois pôles du développement durable et leur forte intrication. Ainsi, la gestion du sanglier montre bien comment l'économique ne peut pas compenser le social : en aucun cas, les indemnisations ne suffisent à limiter un conflit qui se fonde également sur une question de légitimité sociale.

- Ensuite, pour mener une gestion durable du sanglier, il est nécessaire de ne pas s'arrêter aux postures macrosociales mais bien d'aller au-delà, en considérant les « réassemblages sociaux », les nouvelles « associations »d'acteurs visibles dans les postures microsociales (Latour, op. cit.). Il faut notamment être attentif aux enjeux spécifiques dans chaque territoire, qu'ils soient humains ou animaux et savoir décrypter les postures locales des acteurs, à partir notamment de l'histoire de leurs relations. Entre autres, il faut être attentif à l'engagement des acteurs, explicite ou implicite, dans divers contextes d'action. En effet, comme le formule Bossuet (op. cit.), «le déroulement d'un conflit s'inscrit toujours dans un cadre social et spatial historiquement construit, mais dont la scène d'action n'est jamais close. Leurs participants nourrissent des relations polarisées dans et hors de cette scène ».

Pour comprendre les relations entre les protagonistes d'un conflit, il ne faut donc pas se focaliser sur le seul espace-temps de la chasse au sanglier mais prendre en compte l'ensemble des relations au sein du territoire.

Finalement, une gestion durable doit pouvoir articuler des enjeux liés à une problématique générale avec ceux liés au vivre-ensemble local, avec les attentes et les besoins particuliers des individus en présence sur le territoire. 


\section{RÉFÉRENCES BIBLIOGRAPHIQUES}

Baubet E. (1998). Biologie du sanglier en montagne : biodémographie, occupation de l'espace et régime alimentaire. Thèse de doctorat de biologie des populations, Université Claude Bernard, Lyon.

Bobbé S. (1999). Entre sauvage et domestique : le cas du chien errant. Une liminalité bien dérangeante. Ruralia, $\mathrm{n}^{\circ} 5$, p. 119-133.

Boltanski L., Thévenot L. (1991). De la justification. Les économies de grandeur. Paris, Gallimard, nrf Essais.

Bossuet L. (2007). Les conflits du quotidien en milieu rural étude à partir de cinq communes. Géographie, économie, société, 2007/2, vol. 9, p. 141-164.

Callon M. (1986). Eléments pour une socio de la traduction. La domestication des coquilles Saint-Jacques et des marinspêcheurs dans la baie de Saint-Brieuc. L'Année sociologique, 36, p. 169-208 Caron A., Torre A. (2006). Vers une analyse des dimensions négatives de la proximité, Développement durable et territoire. Dossier 7 : «Proximité et environnement », mis en ligne 10 mai 2006, URL : http://developpementdurable.revues .org/document2641.html.

Charlier B. (1999). La défense de l'environnement : entre espace et territoire. Géographie des conflits environnementaux déclanchés en France depuis 1974. Thèse de doctorat de géographie, Université de Pau et des pays de l'Adour.

Dalla Bernardina S. (1996). L'utopie de la nature. Chasseurs, écologistes et touristes. Paris, Imago.

Di Méo G. (2003). Article Territorialité. In Lévy J., Lussault M. (dir.), "Dictionnaire de la géographie et de l'espace des sociétés », Paris, Belin, p. 919.

Debarbieux B. (2003). Article Territoire. In Lévy J., Lussault M. (dir.), « Dictionnaire de la géographie et de l'espace des sociétés », Paris, Belin, p. 910-912.
Decrop G., Dourlens C., Vidal-Naquet P.-A. (2001). L'opacité des scènes locales. In Boyer M., Herzlich G., Maresca B. (coord.), "L'environnement, question sociale; dix ans de recherches pour le ministère de l'Environnement », Paris, éditions Odile Jacob, p. 217-223.

Dziedzicki J.-M. (2001). Gestion des conflits d'aménagement de l'espace : quelle place pour les processus de médiation? Thèse de doctorat en aménagement de l'espace et urbanisme, Université de Tours / CESA.

Friedberg E. (1993). Le Pouvoir et la Règle : Dynamiques de l'action organisée, Paris, Seuil.

Ginelli L., (2008). Chasse-gestion, chasse écologique, chasse durable... Enjeux autour de l'écologisation d'une pratique en crise. Actes du colloque SFER «Chasse, territoires et développement durable. Outils d'analyse, enjeux et perspectives ", Clermont-Ferrand, 25, 26 et 27 mars. Ginelli L., Le Floch S. (2006). Chasséscroisés dans l'espace montagnard. Chasse et renouvellement des liens à l'environnement en Hautes-Pyrénées. Terrain, $\mathrm{n}^{\circ} 47$, p. 123-140.

Guérin M. (chef du groupe de projet Manon) (2005). Conflits d'usage à l'horizon 2020. Quel nouveau rôle pour l'État dans les espaces ruraux et périurbains? Paris, La documentation française.

Gumuchian H., Grasset R., Lajarge R., Roux E. (2003). Les acteurs, ces oubliés du territoire. Paris, Anthropos.

Hirschman A.-O. (1991). Deux siècles de rhétorique réactionnaire. Paris, Fayard, L'espace du politique.

Kaufmann J.-C. (1996). L'entretien compréhensif. Paris, Nathan Universités, série 128.

Latour B. (2006). Changer de société. Refaire de la sociologie. Paris, La Découverte. 
Lecourt A. (2003). Les conflits d'aménagements : analyse théorique et pratique à partir du cas breton. Thèse de doctorat de géographie, Université de Rennes 2 Haute-Bretagne.

Lévy J. (1999). Le tournant géographique. Paris, Belin.

Lussault M. (2009). De la lutte des classes à la lutte des places. Paris, Grasset et Fasquelle.

Lussault M. (2007). L'homme spatial. La construction sociale de l'espace humain. Paris, Seuil, coll. La couleur des idées.

Lussault M. (2003). Article Spatialité. In Lévy J., Lussault M. (dir.), "Dictionnaire de la géographie et de l'espace des sociétés », Paris, Belin, p. 866-868.

Mauz I. (2006a). Introductions, réintroductions : des convergences, par-delà les différences. Natures Sciences et Sociétés, $\mathrm{n}^{\circ} 13, \mathrm{~S} 3 \mathrm{~S} 10$.

Mauz I. (2006b). Préface. Revue de Géographie Alpine, ${ }^{\circ}$ 4, p. 5-6.

Mauz I. (2005). Gens, cornes et crocs. Coéd. Cemagref-Cirad-Ifremer-Inra.

Melé P. (2003). Introduction : conflits, territoires et actions publiques. In Melé P., Larrue C., Rosemberg M. (coord.), «Conflits et territoires », collection perspectives « ville et territoires » $n^{\circ} 6$, Tours, Presses Universitaires François-Rabelais, MSH « ville et territoires », p. 13-32.

Melé P., Larrue C., Rosemberg M. (coord.) (2003). Conflits et territoires. Tours, Presses Universitaires François-Rabelais, Collection perspectives « ville et territoires ».

Micoud A. (1993). Vers un nouvel animal sauvage; le sauvage "naturalisé vivant »? Nature Sciences et Sociétés, vol. $1, \mathrm{n}^{\circ} 3$, p. 202-210.

Micoud A. Bobbé S. (2006). Une gestion durable des espèces animales est-elle possible avec des catégories naturalisées ? Natures Sciences et Sociétés, $\mathrm{n}^{\circ} 14$, p. 32-35.

Mounet C. (2007) Les territoires de l'imprévisible. Conflits, controverses et "vivre ensemble » autour de la gestion de la faune sauvage. Le cas du loup et du sanglier dans les Alpes françaises, Thèse de doctorat de géographie. Université Joseph Fourier, Grenoble I

Mounet C. (2006). Le monde agricole confronté au loup, au sanglier et à leurs partisans : un conflit d'usage et de représentation. Revue de Géographie Alpine, $\mathrm{n}^{\circ}$ 4, p. 89-109.

Perrier-Cornet Ph. (2002). Introduction. La dimension publique de l'espace rural. In Perrier-Cornet Ph. (dir.), « À qui appartient l'espace rural? » La Tour d'Aigues, Éditions de l'Aube, série Bibliothèque des territoires, p. 9-21.

Poinsot Y. (2008). Les enjeux géographiques d'une gestion durable de la faune sauvage en France. Annales de géographie, $\mathrm{n}^{\circ} 663$, p. 26-47.

Poinsot Y., Saldaqui F. (2009). Quelle organisation territoriale pour une gestion durable des sangliers? Un exemple pyrénéen, Mappemonde, $\mathrm{n}^{\circ}$ 94, 2009/2, http://mappemonde.mgm.fr/num22/ articles/art09203.html.

Raynal J.-C. (2004). Pratiques cynégétiques, transformations territoriales et régulations sociales : vers la mise en place d'une gestion concertée des populations de sangliers dans le Sud de la France. Thèse de doctorat de géographie, Université Paul Valery, Montpellier III.

Thévenot L. (2006). L'action au pluriel. Sociologie des régimes d'engagement, Paris, La Découverte.

Thevenot L. (2004). Une science de la vie ensemble dans le monde. Revue MAUSS, $\mathrm{n}^{\circ} 24$, p. 115-126.

Tolon V., Baubet E., Gaulard P., Pasquier J.-J., Hebeisen C., Fischer C., Dobremez J.-F. (2007). Comportement du sanglier en réponse à la pression de chasse. Influence des « réserves» sur son occupation de l'espace. Actes du colloque "Modalités de gestion du sanglier », Reims, 1 et 2 mars. 
Torre A., Aznar O., Bonin M., Caron A., Chia E., Galman M., Guérin M., Jeanneau Ph., Kirat Th., Lefranc Ch., Paoli J.C., Salazar M.I., Thinon P. (2005). Conflits et tensions autour des usages de l'espace dans les territoires ruraux et périurbains. Le cas de la Région Rhône-Alpes et de trois autres zones géographiques françaises. Communication au Symposium international «Territoires et enjeux $d u$ développement régional », Lyon, 9-11 mars.

Torre A., Aznar O., Bonin M., Caron A., Chia E., Galman M., Guérin M., Jeanneau Ph., Kirat Th., Lefranc Ch., Melot R., Paoli J.-C., Salazar M.-I., et
Thinon P. (2006). Conflits et tensions autour des usages de l'espace dans les territoires ruraux et périurbains. Le cas de six zones géographiques françaises. Revue d'Économie Régionale et Urbaine, $\mathrm{n}^{\circ} 3$, p. 415-453.

Traïni C. (2004). Territoires de chasse. Ethnologie française, 2004/2, Tome XXXVII, p. 41-48.

Vourc'h A., Pelosse V. (1988). Chasser dans les Cévennes : un jeu avec l'animal. Aix-en-Provence, CNRS/Edisud.

Weber M. (1971). Économies et sociétés. Les catégories de la sociologie. Paris, Pocket, série Agora. 